\title{
34. EVIDENCE OF MESSINIAN NONMARINE DEPOSITION AT SITE 968 (CYPRUS LOWER SLOPE) ${ }^{1}$
}

\author{
Marie-Madeleine Blanc-Valleron, ${ }^{2}$ Jean-Marie Rouchy, ${ }^{2}$ Catherine Pierre, ${ }^{3}$ Denise Badaut-Trauth, ${ }^{2}$ and Monique Schuler ${ }^{4}$
}

\begin{abstract}
The upper Messinian succession recovered at Ocean Drilling Program Hole 968A on the Cyprus lower slope was generated under brackish conditions (Lago Mare), as deduced from sedimentological and paleontological data. Gypsum that displays marine signatures (stable isotope composition of gypsum and monospecific nannoplankton included in gypsum crystals) is interbedded within this series and interpreted as being reworked from earlier Messinian evaporite deposits, such as those present onshore in Cyprus.
\end{abstract}

\section{INTRODUCTION}

During Ocean Drilling Program (ODP) Leg 160 in the Eastern Mediterranean, drilling at Site 968 (Fig. 1) was performed to investigate the sedimentary and tectonic evolution of the southern Cyprus margin.

Down to $142.5 \mathrm{mbsf}$, the series corresponds to early Pliocene to late Pleistocene nannofossil clay, nannofossil silty clay, and clayey nannofossil ooze (lithostratigraphic Unit I). The upper part of Unit II (142.5-153.8 mbsf), of early Pliocene age (MPL2 zone), corresponds to alternating decimeter-thick bands: one consists of nannofossil ooze and clay and the other of very dark gray clay. The lower part of Unit II (153.8-167 mbsf) corresponds to calcareous clay and silty clay with no biostratigraphic marker present. In Unit III (167-302.7 mbsf), which is composed of calcareous silty clay, silt, and sand, paleontological evidence suggests that this interval may be Miocene in age, with ostracode fauna corresponding to the Lago Mare facies, which is well known in other parts of the Mediterranean (Hsü et al., 1973; Cita et al., 1978, 1990; Rouchy, 1982).

Even though the Sphaeroidinellopsis acme Zone, known in other sites in the Mediterranean and onshore Cyprus in the lowermost part of the Pliocene, was not observed in Hole 968A; therefore, we infer that the Miocene-Pliocene limit corresponds to the marked hiatus between highly carbonated sediments still present in the upper part of Unit II and the more detrital, less carbonated facies in the lower part of Unit II, as in other parts of the Mediterranean. The sediments recovered around this transition indicate that the depositional processes and oceanographic conditions in the region fluctuated significantly.

This report synthesizes X-ray diffraction (XRD) mineralogy, stable isotopes of the gypsum, scanning electron microscopy (SEM) and transmission electron microscopy (TEM) examinations, and palynological data from samples across Units III and II of Hole 968A with the aim of documenting the mineralogy, facies, and paleoenvironments of Messinian to lower Pliocene sediments of the Cyprus lower slope. This report also attempts to constrain the environmental conditions at the Messinian/Pliocene transition. Comparison with the sequences described onshore, in some Cypriot basins, will also be used.

${ }^{1}$ Robertson, A.H.F., Emeis, K.-C., Richter, C., and Camerlenghi, A. (Eds.), 1998. Proc. ODP, Sci. Results, 160: College Station, TX (Ocean Drilling Program).

${ }^{2}$ UA723-CNRS "Processus et Archives Sédimentaires" and Laboratoire de Géologie du MNHN, 43 rue Buffon, 75005 Paris, France. valleron@mnhn.fr

${ }^{3}$ LODYC-CNRS, Université Pierre et Marie Curie, 4 place Jussieu, 75252 Paris Cedex 05, France.

${ }^{4}$ Centre de Géochimie de la Surface-CNRS, 1 rue Blessig, 67084 Strasbourg Cedex,

\section{MATERIAL AND METHODS}

The 132 samples collected from 143.14 to 297.20 mbsf in Hole 968A correspond to the lower part of the early Pliocene marine sequence (18 samples from interval 160-968A-16X-1, 24-25 cm, to $17 \mathrm{X}-2,12-13 \mathrm{~cm})$ and to the inferred Miocene Lago Mare sequence (114 samples from interval 160-968A-17X-2, 35-36 cm, to $34 X-1$, $60-61 \mathrm{~cm})$.

Bulk mineralogy was determined systematically on each sample by calcimetry and XRD. Other methods were applied on selected samples as follows:

$\mathrm{XRD}$ on the carbonate-free residue for clay mineral analysis;

SEM for nannofacies observation;

TEM, sometimes coupled with microprobe analyses, for observation of individual clay particles;

Microscopic examination for observation of sieved fractions;

Isotopic composition of sulfate for gypsum samples;

Rock-Eval pyrolysis; and

Palynological studies.

The carbonate content of each sample was measured on $100 \mathrm{mg}$ of powdered sediment using a manocalcimeter (MCM). Mineralogical analyses were performed by XRD both on bulk samples and carbonate-free residues. Analyses were run with $\mathrm{Cu} \mathrm{K} \alpha$ (Ni filtered) radiation using a Siemens D-500 instrument. Unoriented powders of bulk samples were used to calculate calcite and dolomite percentages by using peak heights and MCM values. After phase identification, semiquantitative estimates of other mineral percentages were calculated. Oriented samples of carbonate-free residues (air dried at room temperature, glycolated, and heat treated at $570^{\circ} \mathrm{C}$ for $90 \mathrm{~min}$ ) were performed according to the procedures outlined in Brindley and Brown (1980). A semiquantitative method, using peak heights, was used to evaluate the relative percentages of illite, kaolinite, chlorite, smectite, illite, mixed-layers, palygorskite, and serpentine, whose amount is reported as recalculated to $100 \%$.

TEM was used to confirm the presence of palygorskite, because the XRD patterns should be considered with caution due to possible confusion with mixed-layer clays. Single-particle analyses were obtained using an energy dispersive spectrometer coupled to a Jeol 100 CX electronic microscope.

Approximately $5 \mathrm{~cm}^{3}$ of sediment was dispersed in distilled water and washed, under running water, through a set of 250-, 125-, and 80$\mu \mathrm{m}$ sieves. Dried residues were examined under a binocular microscope to find the brackish water assemblage identified on board from Sample 160-968A-20X-CC to the bottom of the hole (Shipboard Scientific Party, 1996b). 
Figure 1. Location map of the Leg 160 Hole 968A and Messinian evaporite basins of onshore Cyprus. Bathymetry is shown in meters.

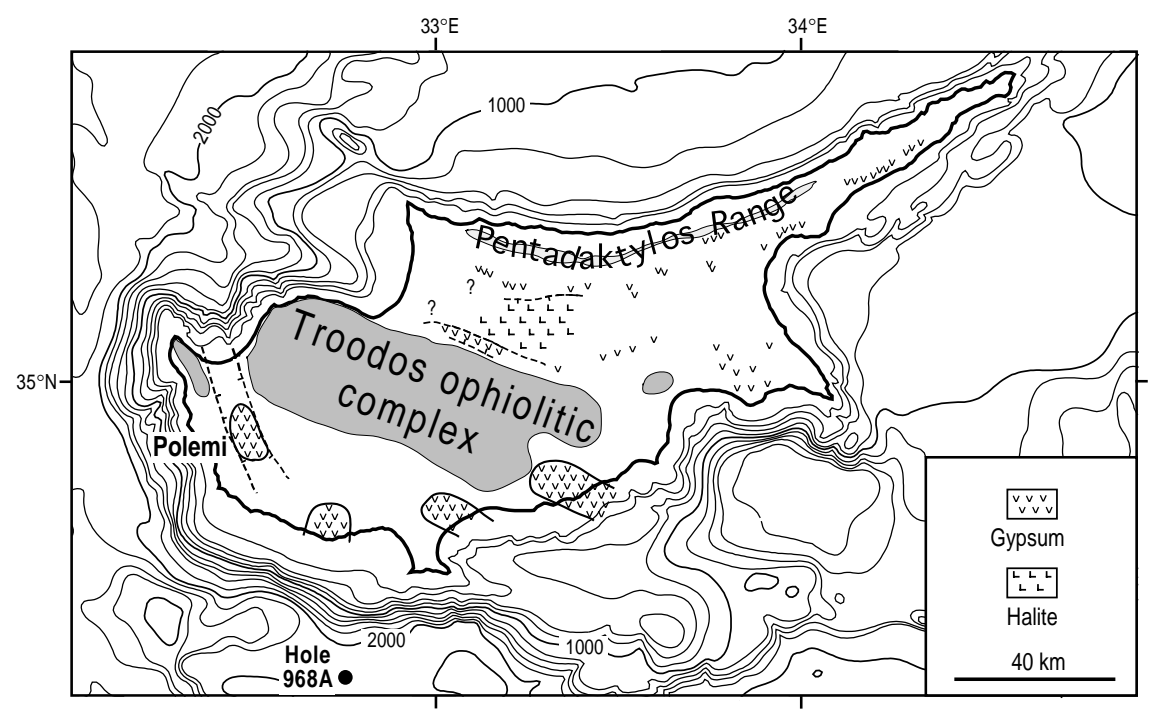

Stable isotope measurements of the gypsum for ${ }^{18} \mathrm{O}$ and ${ }^{34} \mathrm{~S}$ were performed according to the methods used by Pierre and Rouchy (1990) for the study of gypsum samples from ODP Leg 107.

Rock-Eval pyrolysis was performed on bulk sediment samples using a Rock-Eval III-type instrument according to the method described by Espitalié et al. (1977, 1985a, 1985b, 1986). The amount of hydrocarbons $(\mathrm{HC})$ already present in the sample $\left(\mathrm{S}_{1}\right.$ peak in $\mathrm{mg} \mathrm{HC}$ per gram sediment), the amount of $\mathrm{HC}$ generated by pyrolitic degradation of the kerogen during heating of up to $550^{\circ} \mathrm{C}\left(\mathrm{S}_{2}\right.$ peak, in $\mathrm{mg}$ HC per gram sediment), the total organic content (TOC, in weight percent), and the hydrogen index value (HI, quantity of pyrolyzable $\mathrm{HC}$ per gram TOC, in $\mathrm{mg} \mathrm{HC/g} \mathrm{C)} \mathrm{were} \mathrm{determined.}$

Palynological studies were performed according to the methods described in Schuler et al. (1991). Amorphous organic matter (AOM), figured organic matter (FOM), and the main components of FOM were evaluated.

\section{RESULTS AND DISCUSSION Unit II, Upper Part}

The dominant lithologies throughout Unit II are nannofossil ooze, nannofossil clay, calcareous silty clay, and clay. In the upper part of Unit II, between 142.5 and 154 mbsf, the alternating decimeter-thick bands are mainly characterized by their color and carbonate content (Table 1; Fig. 2). In the pale brown to light brown nannofossil ooze and clay, calcite ranges from $41 \%$ to $61 \%$ and dolomite generally ranges from $0 \%$ to $2 \%$, except for the lowermost sample at 160 $968 \mathrm{~A}-17 \mathrm{X}-2,12 \mathrm{~cm}$ (5\% dolomite). The very dark gray clay contains $1 \%-33 \%$ calcite and $0 \%-1 \%$ dolomite.

XRD analyses indicate that clay mineralogy (Table 2) within these two facies is dominated by kaolinite and smectite (Pl. 1), with minor amounts of mixed-layer minerals, palygorskite, illite, and chlorite. Organic matter content is low, even in the dark facies (TOC $\leq 0.9 \%$, see Shipboard Scientific Party, 1996b), mostly reflecting continental input. HI value is low (Table 3), and the FOM is high as compared to the AOM content (Table 4) and corresponds mainly to phytoclasts (fibres, woody fragments).

The kaolinite-smectite assemblage of clays within the lower Pliocene marine setting must correspond to alteration products generated in the hinterland during the warmer and wetter climate of the upper Messinian. This fact is inferred from the intercalations of paleosols within the uppermost Messinian deposits (Lago Mare), associated with the increase of coarse deposition, in some basins from Сy- prus (Rouchy et al., 1980). The dark gray clay bands are composed almost entirely of a kaolinite-smectite mixture, and carbonate corresponds to marine microfossils. These lower Pliocene pelagic sediments display almost the same clay assemblage, but with less chlorite than underlying Messinian sediments (Table 2). We infer that a tectonic and/or relative sea-level change occurred in the late Miocene to early Pliocene time, allowing reworking of alteration products. In the northern part of Cyprus, Messinian compressive tectonics produced the overthrusting of the Pentadaktylos Range onto Messinian evaporites (Baroz et al., 1978), whereas in late Pliocene-early Pleistocene times southern Cyprus underwent its most intense phase of tectonic uplift (Pool and Robertson, 1992).

\section{Unit II, Lower Part}

In the lower part of Unit II, between 153.8 and $167 \mathrm{mbsf}$, light to dark brown calcareous clay alternates with thin layers of dark gray calcareous silty clay. The carbonate fraction is composed of $18 \%-$ $26 \%$ calcite and $2 \%-5 \%$ dolomite. Dolomite is slightly more abundant below $160 \mathrm{mbsf}$, where silty levels are best represented. Smearslide analyses show that inorganic calcite is mostly composed of "rice-grain"-like crystals about 5-20 $\mu \mathrm{m}$ in size in this interval. Quartz, feldspars, clays, amphibole, and serpentine minerals are also present. Chlorite (about 25\%) is more abundant than kaolinite (about $10 \%$ ) in the clay fraction (Table 2), as compared to the upper part of Unit II. FOM becomes less important, as compared to AOM (Table 4 ), and is mainly composed of algal tissues and vegetal cuticles, whereas woody fragments are relatively scarce. Therefore, a sharp change occurs near the transition between Messinian and Pliocene sediments.

The calcareous clays and calcareous silty clays with "rice-grain" calcite facies probably correspond to a transitional environment, such as the one described on top of the Messinian marine shallow-water carbonate deposits of Site 965 (Shipboard Scientific Party, 1996a). These deposits do not contain any indicator of marine or brackish water. The presence of serpentine, which is more abundant than in the upper part of Unit II, confirms an ophiolite origin for the terrigenous input that was presumably derived from Cyprus.

\section{Unit III}

The lithology of Unit III (167-303 mbsf) is dominated by alternating layers of silty clay, silt, and sand. Calcium carbonate values are in the same range as the ones in the basal part of Unit II, from a 
Table 1. Carbonate values for Hole 968A Messinian to early Pliocene samples analyzed.

\begin{tabular}{|c|c|c|c|c|c|}
\hline $\begin{array}{l}\text { Core, section, } \\
\text { interval }(\mathrm{cm})\end{array}$ & $\begin{array}{l}\text { Depth } \\
\text { (mbsf) }\end{array}$ & $\begin{array}{l}\text { Facies/ } \\
\text { color }\end{array}$ & $\begin{array}{l}\text { MCM } \\
(\mathrm{wt} \%)\end{array}$ & $\begin{array}{l}\text { Calcite } \\
(\text { wt } \%)\end{array}$ & $\begin{array}{l}\text { Dolomite } \\
(\mathrm{wt} \%)\end{array}$ \\
\hline \multicolumn{6}{|l|}{ 160-968A- } \\
\hline $16 X-1,24-25$ & 143.14 & & 50 & 49 & 1 \\
\hline $16 X-1,42-43$ & 143.32 & & 17 & 17 & 0 \\
\hline $16 \mathrm{X}-1,59-61$ & 143.49 & & 9 & 9 & 1 \\
\hline $16 X-1,95-97$ & 143.85 & & 13 & 13 & 1 \\
\hline $16 \mathrm{X}-2,100-101$ & 145.40 & & 27 & 26 & 1 \\
\hline $16 \mathrm{X}-3,129-130$ & 147.19 & & 1 & 1 & 0 \\
\hline $16 \mathrm{X}-4,134-135$ & 148.74 & & 43 & 42 & 1 \\
\hline $16 X-5,25-27$ & 149.15 & & 10 & 10 & 0 \\
\hline $16 X-5,43-44$ & 149.33 & & 46 & 45 & 1 \\
\hline $16 X-5,121-122$ & 150.11 & & 43 & 41 & 2 \\
\hline $16 \mathrm{X}-6,72-74$ & 151.12 & & 54 & 52 & 2 \\
\hline $17 X-1,53-54$ & 152.73 & & 55 & 53 & 2 \\
\hline $17 X-1,58-59$ & 152.78 & & 31 & 29 & 2 \\
\hline $17 X-1,98-100$ & 153.18 & & 47 & 46 & 1 \\
\hline $17 X-1,105-107$ & 153.25 & & 34 & 33 & 1 \\
\hline $17 X-1,112-113$ & 153.32 & & 58 & 57 & 1 \\
\hline $17 \mathrm{X}-1,132-134$ & 153.52 & & 62 & 61 & 1 \\
\hline $17 X-2,12-13$ & 153.82 & & 46 & 41 & 5 \\
\hline $17 X-2,35-36$ & 154.05 & & 20 & 18 & 2 \\
\hline $17 X-2,115-116$ & 154.85 & & 21 & 19 & 2 \\
\hline $17 \mathrm{X}-2,140-141$ & 155.10 & & 24 & 23 & 2 \\
\hline $17 X-3,30-31$ & 155.50 & & 20 & 18 & 2 \\
\hline $17 X-3,102-103$ & 156.22 & & 29 & 26 & 2 \\
\hline $17 X-3,135-136$ & 156.55 & & 31 & 29 & 2 \\
\hline $17 X-4,60-61$ & 157.30 & & 27 & 23 & 3 \\
\hline $17 X-4,94-95$ & 157.64 & & 21 & 19 & 2 \\
\hline $17 X-5,60-62$ & 158.80 & & 24 & 21 & 3 \\
\hline $17 X-6,60-61$ & 160.30 & & 31 & 26 & 4 \\
\hline $17 X-6,120-121$ & 160.90 & & 30 & 24 & 5 \\
\hline $17 \mathrm{X}-6,145-146$ & 161.15 & & 28 & 22 & 5 \\
\hline $17 X-7,35-36$ & 161.55 & & 29 & 24 & 5 \\
\hline $18 X-1,35-36$ & 162.15 & & 26 & 22 & 4 \\
\hline $18 X-1,52-53$ & 162.32 & & 26 & 21 & 4 \\
\hline $18 X-2,35-37$ & 163.65 & & 26 & 22 & 3 \\
\hline $18 X-3,65-66$ & 165.45 & & 29 & 26 & 3 \\
\hline $18 X-3,85-86$ & 165.65 & & 28 & 23 & 4 \\
\hline $18 X-4,55-57$ & 166.85 & & 26 & 21 & 4 \\
\hline $18 X-4,80-81$ & 167.10 & & 27 & 21 & 5 \\
\hline $18 X-5,35-36$ & 168.15 & & 32 & 26 & 6 \\
\hline $18 X-5,70-71$ & 168.50 & & 31 & 28 & 3 \\
\hline $18 X-5,120-121$ & 169.00 & & 24 & 20 & 4 \\
\hline $18 X-5,130-131$ & 169.10 & & 23 & 20 & 3 \\
\hline $18 X-6,90-91$ & 170.20 & & 33 & 27 & 6 \\
\hline $18 X-6,103-104$ & 170.33 & & 28 & 24 & 3 \\
\hline $18 X-7,40-41$ & 171.20 & & 24 & 18 & 6 \\
\hline $19 X-1,75-76$ & 172.25 & & 26 & 20 & 5 \\
\hline $19 X-1,88-89$ & 172.38 & & 26 & 23 & 3 \\
\hline $19 X-2,65-66$ & 173.65 & & 23 & 18 & 5 \\
\hline $19 X-2,70-71$ & 173.70 & & 31 & 24 & 6 \\
\hline $19 X-3,35-36$ & 174.85 & & 26 & 20 & 5 \\
\hline $19 X-3,82-83$ & 175.32 & & 34 & 27 & 6 \\
\hline $20 X-1,75-76$ & 181.85 & & 27 & 21 & 5 \\
\hline $20 X-1,80-82$ & 181.90 & & 26 & 21 & 5 \\
\hline 21X-CC, $10-12$ & 190.80 & & 24 & 21 & 3 \\
\hline $22 X-1,45-47$ & 194.45 & & 21 & 18 & 3 \\
\hline $22 X-1,75-76$ & 194.75 & & 22 & 19 & 3 \\
\hline $23 \mathrm{X}-1,26-28$ & 196.26 & & 29 & 25 & 3 \\
\hline $23 X-1,145-146$ & 197.45 & & 26 & 21 & 4 \\
\hline $24 \mathrm{X}-1,44-45$ & 200.84 & & 28 & 25 & 3 \\
\hline $24 X-1,85-86$ & 201.25 & & 29 & 25 & 3 \\
\hline $24 \mathrm{X}-1,120-122$ & 201.60 & & 27 & 23 & 3 \\
\hline $24 X-2,25-26$ & 202.15 & & 29 & 24 & 4 \\
\hline $24 \mathrm{X}-2,125-127$ & 203.15 & & 29 & 25 & 3 \\
\hline $24 X-3,88-89$ & 204.28 & & 29 & 24 & 4 \\
\hline $25 X-1,50-52$ & 210.60 & & 27 & 23 & 3 \\
\hline $25 X-1,70-71$ & 210.80 & & 29 & 24 & 4 \\
\hline $25 \mathrm{X}-1,85-86$ & 210.95 & & 28 & 23 & 4 \\
\hline $25 X-1,93-94$ & 211.03 & & 23 & 20 & 3 \\
\hline $25 \mathrm{X}-1,123-124$ & 211.33 & & 23 & 21 & 3 \\
\hline
\end{tabular}

\begin{tabular}{|c|c|c|c|c|c|}
\hline $\begin{array}{l}\text { Core, section, } \\
\text { interval }(\mathrm{cm})\end{array}$ & $\begin{array}{l}\text { Depth } \\
\text { (mbsf) }\end{array}$ & $\begin{array}{l}\text { Facies/ } \\
\text { color }\end{array}$ & $\begin{array}{l}\text { MCM } \\
(\mathrm{wt} \%)\end{array}$ & $\begin{array}{l}\text { Calcite } \\
(\text { wt } \%)\end{array}$ & $\begin{array}{l}\text { Dolomite } \\
\text { (wt } \%)\end{array}$ \\
\hline $25 X-2,25-26$ & 211.85 & Gypsum & 3 & 2 & 1 \\
\hline $25 X-2,25-26$ & 211.85 & Clayey & 23 & 21 & 2 \\
\hline $25 X-2,55-57$ & 212.15 & Gypsum & 8 & 7 & 1 \\
\hline $25 X-2,55-57$ & 212.15 & Clayey & 21 & 19 & 3 \\
\hline $25 \mathrm{X}-2,120-121$ & 212.80 & Gypsum & 13 & 12 & 1 \\
\hline $25 \mathrm{X}-2,120-121$ & 212.80 & Clayey & 24 & 21 & 3 \\
\hline $25 \mathrm{X}-2,125-126$ & 212.85 & Gypsum & 7 & 7 & 0 \\
\hline $25 \mathrm{X}-\mathrm{CC}, 18-19$ & 213.21 & & 27 & 23 & 3 \\
\hline $25 \mathrm{X}-\mathrm{CC}, 27-28$ & 213.30 & Clayey & 17 & 15 & 2 \\
\hline $25 \mathrm{X}-\mathrm{CC}, 27-28$ & 213.30 & Gypsum & 1 & 1 & 0 \\
\hline $26 \mathrm{X}-1,25-26$ & 219.95 & & 36 & 32 & 3 \\
\hline $26 \mathrm{X}-1,126-128$ & 220.96 & & 33 & 27 & 6 \\
\hline $26 \mathrm{X}-1,136-137$ & 221.06 & & 33 & 28 & 4 \\
\hline $26 X-2,5-6$ & 221.25 & & 56 & 52 & 4 \\
\hline $26 X-2,12-13$ & 221.32 & & 46 & 42 & 3 \\
\hline $26 \mathrm{X}-2,100-101$ & 222.20 & & 20 & 18 & 2 \\
\hline $26 \mathrm{X}-2,123-125$ & 222.43 & & 22 & 20 & 2 \\
\hline $26 \mathrm{X}-3,104-105$ & 223.74 & & 21 & 19 & 2 \\
\hline $26 \mathrm{X}-3,123-125$ & 223.93 & & 23 & 21 & 2 \\
\hline $26 X-4,65-66$ & 224.85 & & 23 & 20 & 3 \\
\hline $27 \mathrm{X}-1,130-132$ & 230.60 & & 23 & 21 & 2 \\
\hline $27 \mathrm{X}-1,141-142$ & 230.71 & & 26 & 23 & 3 \\
\hline $27 \mathrm{X}-2,30-32$ & 231.10 & & 30 & 24 & 5 \\
\hline $27 \mathrm{X}-2,55-56$ & 231.35 & & 22 & 19 & 3 \\
\hline $27 X-3,30-31$ & 232.60 & & 27 & 23 & 4 \\
\hline $27 \mathrm{X}-\mathrm{CC}, 40-41$ & 233.26 & & 25 & 22 & 3 \\
\hline $28 \mathrm{X}-1,62-63$ & 239.52 & & 26 & 21 & 4 \\
\hline $28 \mathrm{X}-1,93-95$ & 239.83 & & 27 & 23 & 4 \\
\hline $28 \mathrm{X}-2,108-110$ & 241.48 & & 28 & 23 & 4 \\
\hline $28 X-3,40-41$ & 242.30 & & 27 & 24 & 3 \\
\hline $28 X-3,60-61$ & 242.50 & & 28 & 24 & 4 \\
\hline $28 \mathrm{X}-3,117-119$ & 243.07 & & 23 & 20 & 3 \\
\hline $28 X-4,74-75$ & 244.14 & & 29 & 20 & 8 \\
\hline $28 \mathrm{X}-4,110-112$ & 244.50 & & 23 & 15 & 8 \\
\hline $28 X-5,63-65$ & 245.53 & & 23 & 20 & 3 \\
\hline $28 \mathrm{X}-6,40-42$ & 246.80 & & 26 & 20 & 5 \\
\hline $29 X-1,65-66$ & 249.25 & & 29 & 22 & 6 \\
\hline $29 X-2,63-64$ & 250.73 & & 26 & 21 & 4 \\
\hline $29 \mathrm{X}-2,130-132$ & 251.40 & & 30 & 24 & 5 \\
\hline $29 \mathrm{X}-3,50-51$ & 252.10 & & 23 & 19 & 4 \\
\hline $29 X-3,130-132$ & 252.90 & & 26 & 21 & 4 \\
\hline $29 X-4,95-96$ & 254.05 & & 28 & 22 & 5 \\
\hline $30 X-1,95-96$ & 259.15 & & 24 & 20 & 4 \\
\hline $30 X-2,95-96$ & 260.65 & & 21 & 15 & 6 \\
\hline $30 X-3,43-45$ & 261.63 & & 21 & 19 & 3 \\
\hline $30 X-4,45-47$ & 263.15 & Pale & 28 & 24 & 3 \\
\hline $30 X-4,45-47$ & 263.15 & Dark & 23 & 20 & 3 \\
\hline $30 X-4,94-95$ & 263.64 & & 22 & 19 & 3 \\
\hline $30 X-5,55-57$ & 264.75 & & 26 & 23 & 3 \\
\hline $30 X-5,103-104$ & 265.23 & & 24 & 21 & 3 \\
\hline $30 X-6,55-57$ & 266.25 & & 26 & 21 & 4 \\
\hline $31 X-1,60-61$ & 268.40 & & 21 & 19 & 2 \\
\hline $31 X-2,90-91$ & 270.20 & & 24 & 21 & 4 \\
\hline $31 X-3,110-111$ & 271.90 & & 23 & 21 & 3 \\
\hline $31 X-4,120-121$ & 273.50 & & 26 & 23 & 3 \\
\hline $32 X-1,50-51$ & 277.90 & & 27 & 22 & 4 \\
\hline $32 X-2,90-91$ & 279.80 & & 28 & 24 & 3 \\
\hline $32 \mathrm{X}-3,120-121$ & 281.60 & & 31 & 25 & 5 \\
\hline $32 \mathrm{X}-4,130-131$ & 283.20 & & 29 & 24 & 4 \\
\hline $32 X-5,100-101$ & 284.40 & & 28 & 23 & 4 \\
\hline $32 X-6,70-71$ & 285.60 & & 26 & 18 & 7 \\
\hline $33 X-1,35-36$ & 287.35 & & 29 & 24 & 4 \\
\hline $33 \mathrm{X}-2,85-86$ & 289.35 & & 26 & 22 & 3 \\
\hline $33 X-3,135-137$ & 291.35 & & 27 & 22 & 5 \\
\hline $33 \mathrm{X}-4,130-131$ & 292.80 & & 27 & 23 & 3 \\
\hline $33 \mathrm{X}-5,125-126$ & 294.25 & & 26 & 22 & 3 \\
\hline $33 X-6,125-126$ & 295.75 & & 23 & 21 & 3 \\
\hline $34 \mathrm{X}-1,60-61$ & 297.20 & & 27 & 19 & 7 \\
\hline
\end{tabular}

Note: Heterogeneous facies ( $\mathrm{cf}$ facies/color column) have been split before analysis.

minimum of $22 \%$ to a maximum of $35 \%$. Exceptions are some low values corresponding to gypsum levels and higher values around 220 mbsf. XRD analyses indicate the presence of calcite, quartz, feldspar, dolomite, clay, amphibole, and serpentine. The clay assemblage is similar to the one at the base of Unit II (Table 2; Pls. 1, 2): serpentine content is quite variable $(10 \%-40 \%)$, with the higher percentages in the clay fraction corresponding to the coarser levels. These sediments are characterized in smear-slide analysis by their relatively low nannofossil content $(0 \%-10 \%)$. Most of the nannofossil assemblages correspond to reworked taxa. Nevertheless, brackish to fresh water (Lago Mare) or even a continental environment (pulmonate gastro- pod at Sample 160-968A-26X-3, 123-125 cm) can be inferred from Ammonia sp., Cyprideis sp., and gastropod faunas (Table 5).

Between 167 and 194 mbsf, alternating intervals of centimeter- to decimeter-thick silty clay, silt, and sand occur. Colors vary from gray, olive-gray, and dark greenish gray to dark gray. Sediments are characterized by relatively low clay percentages $(\sim 30 \%)$ and the presence of dolomite. Calcite ranges from $18 \%$ to $28 \%$ (same as above) and dolomite from $3 \%$ to $6 \%$ (slightly higher).

From 194 to 239 mbsf, the lithology is dominated by olive-gray, dark gray, grayish brown, and reddish calcareous silty clay. Calcite ranges from $18 \%$ to $32 \%$ (with some higher values, $52 \%$ and $42 \%$, 


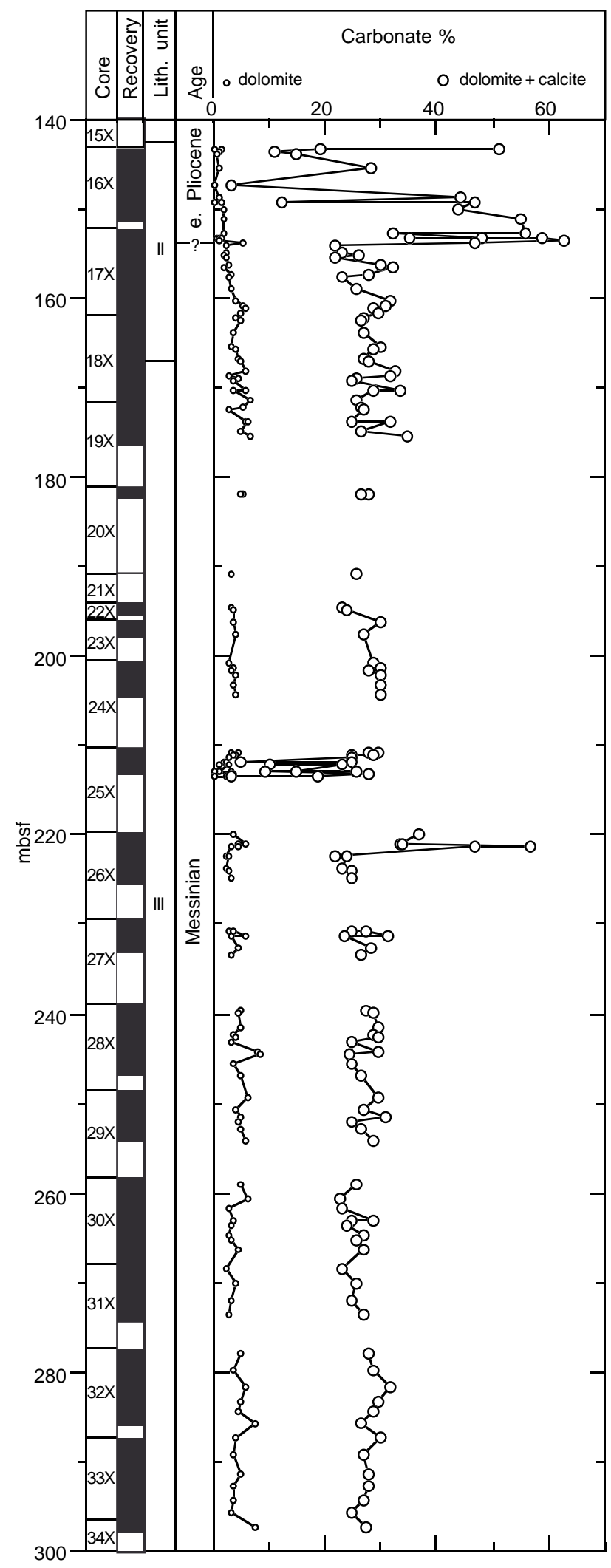

Figure 2. Carbonate content of the studied part of Hole 968A.
Table 2. Clay mineral composition of Hole 968A Messinian to early Pliocene samples analyzed.

\begin{tabular}{|c|c|c|c|c|c|c|c|c|}
\hline $\begin{array}{l}\text { Core, section, } \\
\text { interval }(\mathrm{cm})\end{array}$ & $\begin{array}{l}\text { Depth } \\
\text { (mbsf) }\end{array}$ & $\mathrm{Sm}$ & $\begin{array}{c}\text { Mixed } \\
\text { layer }\end{array}$ & Palygo & Illite & Serp & Kaol & Chlor \\
\hline \multicolumn{9}{|l|}{$160-968 \mathrm{~A}-$} \\
\hline $16 \mathrm{X}-1,24-25$ & 143.14 & 30 & 5 & 5 & 10 & 5 & 35 & 10 \\
\hline $16 \mathrm{X}-2,100-101$ & 145.40 & 25 & 5 & 5 & 10 & 5 & 40 & 10 \\
\hline $16 \mathrm{X}-3,129-130$ & 147.19 & 20 & 5 & 5 & 5 & 10 & 45 & 10 \\
\hline $16 \mathrm{X}-4,134-135$ & 148.74 & 20 & 5 & 5 & 10 & 5 & 45 & 10 \\
\hline $16 X-5,25-27$ & 149.15 & 30 & 5 & 5 & 5 & 10 & 40 & 5 \\
\hline $16 \mathrm{X}-6,72-74$ & 151.12 & 15 & 5 & 5 & 15 & 5 & 45 & 10 \\
\hline $17 \mathrm{X}-2,12-13$ & 153.82 & 20 & 5 & 5 & 15 & 15 & 20 & 20 \\
\hline $17 \mathrm{X}-2,35-36$ & 154.05 & 20 & 10 & 10 & 15 & 10 & 10 & 25 \\
\hline $18 \mathrm{X}-2,35-37$ & 163.65 & 25 & 5 & 10 & 15 & 10 & 10 & 25 \\
\hline $19 \mathrm{X}-2,70-71$ & 173.70 & 20 & 0 & 5 & 5 & 35 & 10 & 25 \\
\hline $20 \mathrm{X}-1,80-82$ & 181.90 & 25 & 5 & 5 & 15 & 20 & 10 & 20 \\
\hline $21 \mathrm{X}-\mathrm{CC}, 10-12$ & 190.80 & 30 & 5 & 5 & 15 & 15 & 10 & 20 \\
\hline $22 \mathrm{X}-1,45-47$ & 194.45 & 20 & 5 & 5 & 10 & 30 & 15 & 20 \\
\hline $23 \mathrm{X}-1,26-28$ & 196.26 & 20 & 5 & 10 & 15 & 25 & 5 & 20 \\
\hline $24 \mathrm{X}-1,120-122$ & 201.60 & 25 & 5 & 10 & 15 & 20 & 5 & 20 \\
\hline $24 \mathrm{X}-2,125-127$ & 203.15 & 20 & 5 & 10 & 10 & 25 & 10 & 20 \\
\hline $25 \mathrm{X}-1,50-52$ & 210.60 & 25 & 5 & 10 & 10 & 20 & 10 & 20 \\
\hline $25 X-2,55-57$ & 212.15 & 25 & 5 & 10 & 15 & 15 & 10 & 20 \\
\hline $26 \mathrm{X}-1,136-137$ & 221.06 & 25 & 5 & 5 & 20 & 15 & 5 & 25 \\
\hline $26 \mathrm{X}-2,123-125$ & 222.43 & 35 & 5 & 5 & 15 & 10 & 10 & 20 \\
\hline $26 \mathrm{X}-3,123-125$ & 223.93 & 35 & 5 & 5 & 15 & 10 & 10 & 20 \\
\hline $27 \mathrm{X}-1,130-132$ & 230.60 & 35 & 5 & 5 & 15 & 10 & 5 & 25 \\
\hline $27 \mathrm{X}-2,30-32$ & 231.10 & 30 & 5 & 5 & 10 & 10 & 10 & 30 \\
\hline $28 \mathrm{X}-1,93-95$ & 239.83 & 30 & 5 & 5 & 15 & 10 & 10 & 25 \\
\hline $28 \mathrm{X}-4,110-112$ & 244.50 & 15 & 0 & 5 & 10 & 40 & 10 & 20 \\
\hline $28 X-5,63-65$ & 245.53 & 25 & 0 & 5 & 5 & 35 & 10 & 20 \\
\hline $28 \mathrm{X}-6,40-42$ & 246.80 & 20 & 0 & 5 & 5 & 40 & 10 & 20 \\
\hline $29 \mathrm{X}-2,130-132$ & 251.40 & 20 & 0 & 5 & 20 & 20 & 10 & 25 \\
\hline $29 \mathrm{X}-3,130-132$ & 252.90 & 20 & 0 & 5 & 10 & 35 & 10 & 20 \\
\hline $30 X-4,45-47$ & 263.15 & 30 & 5 & 5 & 15 & 10 & 10 & 25 \\
\hline $30 X-5,55-57$ & 264.75 & 25 & 5 & 5 & 15 & 15 & 10 & 25 \\
\hline $30 X-6,55-57$ & 266.25 & 30 & 5 & 5 & 15 & 10 & 10 & 25 \\
\hline $31 X-2,90-91$ & 270.20 & 30 & 5 & 5 & 15 & 10 & 10 & 25 \\
\hline $31 \mathrm{X}-4,120-121$ & 273.50 & 30 & 5 & 5 & 15 & 10 & 10 & 25 \\
\hline $32 X-1,50-51$ & 277.90 & 25 & 5 & 5 & 20 & 15 & 5 & 25 \\
\hline $32 \mathrm{X}-3,120-121$ & 281.60 & 20 & 5 & 5 & 20 & 15 & 5 & 30 \\
\hline $33 \mathrm{X}-1,35-36$ & 287.35 & 30 & 5 & 5 & 15 & 15 & 5 & 25 \\
\hline $33 X-5,125-126$ & 294.25 & 30 & 5 & 5 & 15 & 10 & 5 & 30 \\
\hline $34 \mathrm{X}-1,60-61$ & 297.20 & 15 & 5 & 5 & 15 & 20 & 10 & 30 \\
\hline
\end{tabular}

Notes: Smectite (Sm), mixed-layer clays, palygorskite (Palygo), illite, serpentine (Serp), kaolinite (Kaol), and chlorite (Chl) in relative percent of the $<40-\mu \mathrm{m}$ fraction.

corresponding to pale, fine-grained facies associated with more detritic sandy layers), and dolomite ranges from $2 \%$ to $6 \%$. Thin (up to $2 \mathrm{~cm}$ thick), white gypsum-rich silt beds (gypsites) alternate with the grayish brown silty clay in the interval between 211 and $213 \mathrm{mbsf}$. A thicker (13 cm thick) alabastrine gypsum bed is also present (interval 160-968A-25X-CC, 26-40 cm).

Several gypsum samples from millimeter- to centimeter-thick levels have been observed by SEM (Pl. 3). Gypsum crystals are heterogranular and usually range in size from 10 to $200 \mu \mathrm{m}$. The samples include, or are mixed with, monospecific nannofossils as described in balatino levels from upper Messinian levels in the Mediterranean (Rouchy, 1982).

Three gypsum samples were selected in Core 160-968A-25X for analysis of their oxygen and sulfur stable isotope compositions. The oxygen and sulfur isotope compositions of sulfate represent wellknown tracers of the origin of the sulfate in solution, which precipitates evaporites. The numerous isotopic data available on Messinian gypsum and anhydrite (Pierre, 1974, 1982; Pierre and Rouchy, 1990; Rouchy and Pierre, 1979; Pierre and Fontes, 1978) have demonstrated that sulfate evaporites precipitated mostly from the oceanic sulfate reservoir throughout Messinian time in the Mediterranean, although large differences in the redox regime were occurring, and especially in the most restricted parts of the basins.

At Site 968, the $\delta$ values of the gypsum are also characteristic of marine Tertiary sulfate (Table 6 ). $\delta^{18} \mathrm{O}$ values are very homogeneous (16.8-17.1) and very similar to the ones measured in the present-day selenitic gypsum from the solar ponds of Salin-de-Giraud (Pierre and Fontes, 1982). $\delta^{34} \mathrm{~S}$ values display significant variations from $22.7 \%$ o (Sample 160-968A-25X-2. $125 \mathrm{~cm}$ ) and 22.3\%o (Sample 160-968A- 
Table 3. Rock-Eval data for Hole 968A Messinian to early Pliocene analyzed samples.

\begin{tabular}{lrrrrrr}
\hline $\begin{array}{c}\text { Core, section, } \\
\text { interval }(\mathrm{cm})\end{array}$ & $\begin{array}{c}\text { Depth } \\
\text { (mbsf) }\end{array}$ & $\Sigma$ Carb. & TOC & $\mathrm{S}_{1}$ & $\mathrm{~S}_{2}$ & $\mathrm{HI}$ \\
\hline 160-968A- & & & & & & \\
16X-1, 59-61 & 143.49 & 9 & 0.09 & 0.03 & 0.03 & \\
16X-1, 95-97 & 143.85 & 13 & 0.19 & 0.18 & 7.72 & \\
16X-3, 129-130 & 147.19 & 1 & 0.35 & 0.05 & 0.17 & 48 \\
16X-5, 25-27 & 149.15 & 46 & 0.21 & 0.04 & 0.06 & \\
17X-1, 105-107 & 153.25 & 34 & 0.10 & 0.03 & 0.03 & \\
17X-5, 60-62 & 158.80 & 24 & 0.13 & 0.03 & 0.05 & \\
18X-4, 55-57 & 166.85 & 25 & 0.14 & 0.03 & 0.07 & \\
20X-1, 80-82 & 181.90 & 25 & 0.14 & 0.02 & 0.06 & \\
26X-1, 126-128 & 220.96 & 32 & 0.40 & 0.06 & 0.14 & 35 \\
30X-3, 43-45 & 261.63 & 21 & 0.14 & 0.04 & 0.06 & \\
& & & & & &
\end{tabular}

Notes: Carbonates ( $\Sigma$ Carb.) and total organic carbon (TOC) in weight percent of bulk sediment. Units for Rock-Eval parameters are as follows: $\mathrm{S}_{1}$ (mg HC/g sediment), $\mathrm{S}_{2}$ (mg HC/g sediment), and $\mathrm{HI}=\mathrm{S}_{2} / \mathrm{TOC}$ ( $\mathrm{mg} \mathrm{HC} / \mathrm{g}$ TOC).

Table 4. Quantification of amorphous and figured organic matter within the total "organic matter" residue of Messinian to early Pliocene analyzed samples.

\begin{tabular}{|c|c|c|c|c|c|}
\hline $\begin{array}{l}\text { Core, section, } \\
\text { interval }(\mathrm{cm})\end{array}$ & $\begin{array}{l}\text { Depth } \\
\text { (mbsf) }\end{array}$ & $\begin{array}{c}\Sigma \text { Carb. } \\
\text { (wt } \%)\end{array}$ & $\begin{array}{c}\text { "OM" } \\
(\mathrm{mL} / 10 \mathrm{~g})\end{array}$ & $\begin{array}{c}\mathrm{AOM} \\
(\mathrm{mL} / 10 \mathrm{~g})\end{array}$ & $\begin{array}{c}\text { FOM } \\
(\mathrm{mL} / 10 \mathrm{~g})\end{array}$ \\
\hline \multicolumn{6}{|l|}{ 160-968A- } \\
\hline $16 \mathrm{X}-1,59-61$ & 143.49 & 9 & 0.21 & 0.02 & 0.11 \\
\hline $16 \mathrm{X}-1,95-97$ & 143.85 & 13 & 0.19 & 0.02 & 0.13 \\
\hline $16 X-5,25-27$ & 149.15 & 10 & 0.25 & 0.03 & 0.20 \\
\hline $16 X-6,72-74$ & 151.12 & 54 & 0.00 & 0.00 & 0.00 \\
\hline $17 \mathrm{X}-1,98-100$ & 153.18 & 47 & 0.19 & 0.04 & 0.11 \\
\hline $17 \mathrm{X}-1,105-107$ & 153.25 & 34 & 0.19 & 0.11 & 0.04 \\
\hline $17 \mathrm{X}-1,132-134$ & 153.52 & 62 & 0.18 & 0.06 & 0.12 \\
\hline $17 X-5,60-62$ & 158.80 & 24 & 0.16 & 0.10 & 0.06 \\
\hline $18 X-2,35-37$ & 163.65 & 25 & 0.07 & 0.04 & 0.03 \\
\hline $18 X-4,55-57$ & 166.85 & 25 & 0.18 & 0.11 & 0.05 \\
\hline $20 \mathrm{X}-1,80-82$ & 181.90 & 25 & 0.28 & 0.06 & 0.20 \\
\hline 21X-CC, $10-12$ & 190.80 & 24 & 0.22 & 0.07 & 0.02 \\
\hline $24 \mathrm{X}-1,120-122$ & 201.60 & 26 & 0.09 & 0.06 & 0.03 \\
\hline $25 \mathrm{X}-1,50-52$ & 210.60 & 26 & 0.15 & 0.00 & 0.03 \\
\hline $26 \mathrm{X}-1,126-128$ & 220.96 & 32 & 0.42 & 0.17 & 0.04 \\
\hline $27 \mathrm{X}-1,130-132$ & 230.60 & 23 & 0.11 & 0.07 & 0.02 \\
\hline $28 \mathrm{X}-2,108-110$ & 241.48 & 28 & 0.21 & 0.02 & 0.02 \\
\hline $29 \mathrm{X}-2,130-132$ & 251.40 & 29 & 0.16 & 0.02 & 0.06 \\
\hline $30 X-3,43-45$ & 261.63 & 21 & 0.19 & 0.05 & 0.05 \\
\hline $33 X-3,135-137$ & 291.35 & 26 & 0.15 & 0.06 & 0.03 \\
\hline
\end{tabular}

Note: $\mathrm{AOM}=$ amorphous organic matter, $\mathrm{FOM}$ = figured organic matter, "OM" = total "organic matter" residue.

$25 \mathrm{X}-\mathrm{CC}, 27 \mathrm{~cm}$ ) to $20.8 \%$ (Sample 160-968A-25X-2, $25 \mathrm{~cm}$ ). At this level, macroscopic and microscopic observations revealed that gypsum is partly composed of sulfate generated from sulfide oxidation (Pl. 3, Fig. 3). The $1.5 \% \%_{-} 2 \%$ o decrease in the $\delta^{34} \mathrm{~S}$ value, accompanied by a very weak decrease in the $\delta^{18} \mathrm{O}$ value, can be related to the contribution of a fraction of reoxidized bacterial sulfide depleted in ${ }^{34} \mathrm{~S}$.

The interval between 239 and 260.7 mbsf consists of fine gray to dark gray sand and calcareous silty clay, containing centimeter- to decimeter-thick mud beds. Some of these beds are graded and display erosional bases. Calcite ranges from $15 \%$ to $24 \%$ and dolomite from $3 \%$ to $6 \%$.

The interval between 260.5 and 286.5 mbsf contains dark gray calcareous clay with centimeter-thick silty layers. Calcite ranges from $18 \%$ to $25 \%$ and dolomite from $2 \%$ to $7 \%$. The gastropod found at 264.75 mbsf (Table 5) is a Brotia (Melanidae), corresponding to a supralittoral environment.

From 286.5 to the base of the unit at $303 \mathrm{mbsf}$, gray calcareous silty clay and calcareous silty sand occur. Calcite ranges from $19 \%$ to $24 \%$ and dolomite from $3 \%$ to $7 \%$.

Unit III corresponds to a detrital facies where products derived from the Troodos ophiolite, during and after its uplift, are abundant. The detrital gypsum may also have been derived from a paleoslope
Table 5. Main biological components of the Lago Mare facies within analyzed samples.

\begin{tabular}{|c|c|c|c|c|c|}
\hline $\begin{array}{l}\text { Core, section, } \\
\text { interval }(\mathrm{cm})\end{array}$ & $\begin{array}{l}\text { Depth } \\
\text { (mbsf) }\end{array}$ & $\begin{array}{l}\Sigma \text { Carb. } \\
\text { (wt } \%)\end{array}$ & Gastropoda & Cyprideis & Ammonia \\
\hline \multicolumn{6}{|l|}{ 160-968A- } \\
\hline $19 X-2,70-71$ & 173.70 & 30 & & + & + \\
\hline $20 \mathrm{X}-1,80-82$ & 181.90 & 25 & & & \\
\hline 21X-CC, 10-12 & 190.80 & 24 & & + & + \\
\hline $22 X-1,45-47$ & 194.45 & 21 & & + & + \\
\hline $23 \mathrm{X}-1,26-28$ & 196.26 & 28 & & + & + \\
\hline $24 \mathrm{X}-1,120-122$ & 201.60 & 26 & & & \\
\hline $24 \mathrm{X}-2,125-127$ & 203.15 & 28 & & + & \\
\hline $25 \mathrm{X}-1,50-52$ & 210.60 & 26 & & + & \\
\hline $25 X-2,55-57$ & 212.15 & 21 & & & + \\
\hline $26 \mathrm{X}-1,136-137$ & 221.06 & 32 & & & \\
\hline $26 \mathrm{X}-2,123-125$ & 222.43 & 22 & & & \\
\hline $26 \mathrm{X}-3,123-125$ & 223.93 & 23 & + & & \\
\hline $27 \mathrm{X}-1,130-132$ & 230.60 & 23 & & + & \\
\hline $27 \mathrm{X}-2,30-32$ & 231.10 & 30 & & + & + \\
\hline $28 \mathrm{X}-1,93-95$ & 239.83 & 27 & & + & \\
\hline $28 \mathrm{X}-4,110-112$ & 244.50 & 23 & & & + \\
\hline $28 X-5,63-65$ & 245.53 & 23 & & & \\
\hline $28 X-6,40-42$ & 246.80 & 25 & & & \\
\hline $29 \mathrm{X}-2,130-132$ & 251.40 & 29 & & & \\
\hline $29 X-3,130-132$ & 252.90 & 25 & & & \\
\hline $30 X-4,45-47$ & 263.15 & 25 & + & + & \\
\hline $30 X-5,55-57$ & 264.75 & 25 & + & + & \\
\hline $30 X-6,55-57$ & 266.25 & 25 & & + & \\
\hline $31 X-2,90-91$ & 270.20 & 24 & & & \\
\hline $31 X-4,120-121$ & 273.50 & 25 & & & \\
\hline $32 \mathrm{X}-1,50-51$ & 277.90 & 26 & & & \\
\hline $32 \mathrm{X}-3,120-121$ & 281.60 & 30 & & & \\
\hline $33 \mathrm{X}-1,35-36$ & 287.35 & 28 & & & \\
\hline $33 X-5,125-126$ & 294.25 & 25 & & & \\
\hline $34 X-1,60-61$ & 297.20 & 26 & & & \\
\hline
\end{tabular}

Note: $+=$ present.

within southern Cyprus (or present offshore areas), where gypsum locally developed in small silled basins during the upper Messinian (Orszag-Sperber et al., 1980; Robertson et al., 1995).

\section{CONCLUSION}

The main conclusion of this study is that the sedimentary paleoenvironments of the upper Messinian facies of the Cyprus lower slope are consistent with previous studies of Lago Mare facies in the Eastern and Western Mediterranean.

Gypsum present within this offshore series displays a marine isotope signature and incorporates marine nannofossils. These facts are not consistent with the brackish conditions inferred for the Lago Mare interval. We interpret the gypsum as being reworked from basins of onshore Cyprus, or in present offshore areas, exposed in Messinian times by evaporitic drawdown. As an example, the upper evaporitic unit in the Polemi basin is truncated by an erosional surface overlain by the Lago Mare facies (Orszag-Sperber et al., 1989).

\section{ACKNOWLEDGMENTS}

The authors thank A.-M. Brunet, P. Clement, M. Destarac, and M. Tamby for technical assistance; D. Merle for paleontological determinations of gastropods; and M.-T. Vénec-Peyre, A. Foucault, and F. Mélières for scientific discussion.

Financial support for this study was provided by the Institut National des Sciences de l'Univers (INSU grant N 963905 for "Marine Geosciences" program).

\section{REFERENCES}

Baroz, F., Bernouilli, D., Biju-Duval, B., Bizon, G., Bizon, J.J., and Letouzey, J., 1978. Correlations of the Neogene formations of the Florence Rise and of northern Cyprus: paleogeographic and structural implications. In Hsü, K., Montadert, L., et al., Init. Repts. DSDP, 42: Washington (U.S. Govt. Printing Office), 903-926. 
Brindley, G.W., and Brown, G. (Eds.), 1980. Crystal Structures of Clay Minerals and Their X-ray Identification. Mineral. Soc. London Monogr., 5.

Cita, M.B., Santambrogio, S., Melillo, B., and Rogate, F., 1990. Messinian paleoenvironments: new evidence from the Tyrrhenian Sea (ODP Leg 107). In Kastens, K.A., Mascle, J., et al., Proc. ODP, Sci. Results, 107 : College Station, TX (Ocean Drilling Program), 211-227.

Cita, M.B., Wright, R.C., Ryan, W.B.F., and Longinelli, A., 1978. Messinian paleoenvironments. In Hsü K.J., Montadert, L., et al., Init. Repts. DSDP, 42 (Pt. 1): Washington (U.S. Govt. Printing Office), 1003-1035.

Espitalié, J., Deroo, G., and Marquis, F., 1985a. La pyrolyse Rock-Eval et ses applications, Partie I. Rev. Inst. Fr. Pet., 40/5:563-579.

, 1985b. La pyrolyse Rock-Eval et ses applications, Partie II. Rev. Inst. Fr. Pet., 40/6:755-784.

, 1986. La pyrolyse Rock-Eval et ses applications, Partie III. Rev. Inst. Fr. Pet., 41:73-89.

Espitalié, J., Laporte, J.L., Leplat, P., Madec, M., Marquis, F., Paulet, J., and Boutefeu, A., 1977. Méthode rapide de caractérisation des roches mères, de leur potentiel pétrolier et de leur degré d'évolution. Rev. Inst. Fr. Pet., 32:23-42.

Hsü, K.J., Cita, M.B., and Ryan, W.B.F., 1973. The origin of the Mediterranean evaporites. In Ryan, W.B.F., Hsü, K.J., et al., Init. Repts. DSDP, 13 (Pt. 2): Washington (U.S. Govt. Printing Office), 1203-1231.

Orszag-Sperber, F., Rouchy, J.M., Bizon, G., Bizon, J.J., Cravatte J., and Muller, C., 1980. La sédimentation messinienne dans le bassin de Polemi (Chypre). Geol. Mediterr., 7:93-102.

Orszag-Sperber, F., Rouchy, J.M., and Elion, P., 1989. The sedimentary expression of regional tectonic events during the Miocene-Pliocene transition in the southern Cyprus basins. Geol. Mag., 126:291-299.

Pierre, C., 1974. Contribution à l'étude sédimentologique et isotopique des évaporites messiniennes de la Méditerranée: implications géodynamiques [thesis]. Univ. Paris VI.

1982. Teneurs en isotopes stables $\left({ }^{18} \mathrm{O},{ }^{2} \mathrm{H},{ }^{13} \mathrm{C},{ }^{34} \mathrm{~S}\right)$ et conditions de genèse des évaporites marines: application à quelques milieux actuels et au Messinien de Méditerranée [Doct. thesis]. Univ. Paris-Sud Orsay.

Pierre, C., and Fontes, J.C., 1978. Isotope composition of Messinian sediments from the Mediterranean sea as indicators of paleoenvironments and diagenesis. In Hsü, K.J., Montadert, L., et al., Init. Repts. DSDP, 42: Washington (U.S. Govt. Printing Office), 635-650.
1982. Etude isotopique des saumures et des gypses des marais salants de Salin-de-Giraud (Sud de la France). Geol. Mediterr., 4:479486.

Pierre, C., and Rouchy, J.M., 1990. Sedimentary and diagenetic evolution of Messinian evaporites in the Tyrrhenian Sea (ODP Leg 107, Sites 652, 653, and 654): petrographic, mineralogical, and stable isotope records. In Kastens, K.A., Mascle, J., et al., Proc. ODP, Sci. Results, 107: College Station, TX (Ocean Drilling Program), 187-210.

Poole, A.J., and Robertson, A.H.F., 1992. Quaternary uplift and sea-level change at an active plate boundary, Cyprus. J. Geol. Soc. London, 148:909-921.

Robertson, A.H.F., Eaton, S.E., Follows, E.J., and Payne, A.S., 1995. Sedimentology and depositional processes of Miocene evaporites from Cyprus. Terra Nova, 7:233-254.

Rouchy, J.M., 1982. La genèse des évaporites messiniennes de Méditerranée. Mem. Mus. Nat. Hist. Nat., 50:1-267.

Rouchy, J.M., and Pierre, C., 1979. Données sédimentologiques et isotopiques sur les gypses des séries évaporitiques messiniennes d'Espagne méridionale et de Chypre. Rev. Geogr. Phys.Geol. Dyn., 21:267-280.

Rouchy, J.M.-, Orszag-Sperber, F., Bizon, G., and Bizon, J.-J., 1980. Mise en évidence d'une phase d'émersion fini-messinienne dans le bassin de Pissouri (Chypre): une modalité de passage Miocène-Pliocène en Méditerranée orientale.C. R.Acad. Sci. Ser. 2, 291:729-732.

Schuler, M., Blanc-Valleron, M.M., Gely, J.P., and Ansart, M., 1991. Matière organique et palynofaciès de l'Oligocène inférieur évaporitique du bassin de Mulhouse (France). Palynosciences, 1:41-58.

Shipboard Scientific Party, 1996a. Site 965. In Emeis, K.-C., Robertson, A.H.F., Richter, C., et al., Proc. ODP, Init. Repts., 160: College Station, TX (Ocean Drilling Program), 125-153.

1996b. Site 968. In Emeis, K.-C., Robertson, A.H.F., Richter, C., et al., Proc. ODP, Init. Repts., 160: College Station, TX (Ocean Drilling Program), 289-333.

Date of initial receipt: 7 January 1997

Date of acceptance: 22 June 1997

Ms 160SR-001

Table 6. Simplified lithologic description and stable isotope composition of gypsum in ODP Hole 968A.

\begin{tabular}{|c|c|c|c|c|c|c|c|}
\hline $\begin{array}{l}\text { Core, section, } \\
\text { interval }(\mathrm{cm})\end{array}$ & $\begin{array}{l}\text { Depth } \\
\text { (mbsf) }\end{array}$ & Lithologic description & $\begin{array}{l}\text { MCM } \\
\text { (wt \%) }\end{array}$ & $\begin{array}{l}\text { Calcite } \\
(\mathrm{wt} \%)\end{array}$ & $\begin{array}{c}\text { Dolomite } \\
\text { (wt } \%)\end{array}$ & $\begin{array}{c}\delta^{18} \mathrm{O}(\% 0) \\
\text { SMOW }\end{array}$ & $\begin{array}{c}\delta^{34} \mathrm{~S}(\% \circ) \\
\mathrm{CDT}\end{array}$ \\
\hline \multicolumn{8}{|l|}{ 160-968A- } \\
\hline $25 \mathrm{X}-2,25-26$ & 211.85 & Limonite-rich (orange stained), $\mathrm{mm}$ thick gypsum level & 3 & 2 & 1 & 16.8 & 20.8 \\
\hline $25 X-2,125-126$ & 212.85 & White gypsum, part of $\mathrm{cm}$-thick level & 7 & 7 & 0 & 17.1 & 22.7 \\
\hline $25 \mathrm{X}-\mathrm{CC}, 27-28$ & 213.30 & White gypsum, part of dm-thick level & 1 & 1 & 0 & 17.0 & 22.3 \\
\hline
\end{tabular}

Note: $\mathrm{SMOW}=$ Standard Mean Ocean Water, CDT = Cañon Diablo Troilite. 


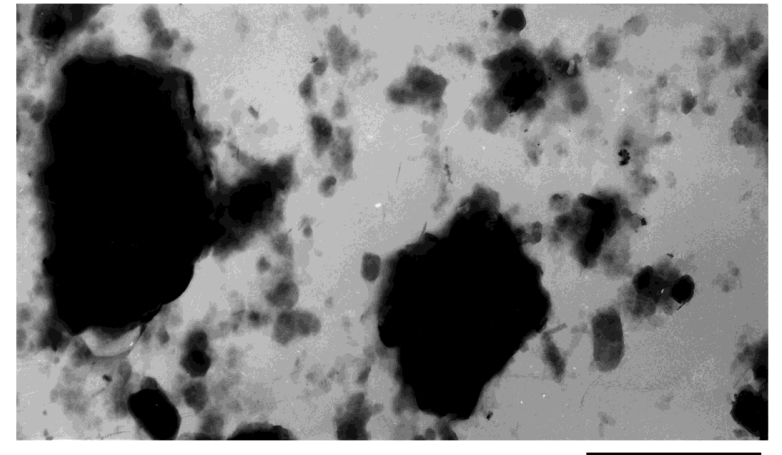

1

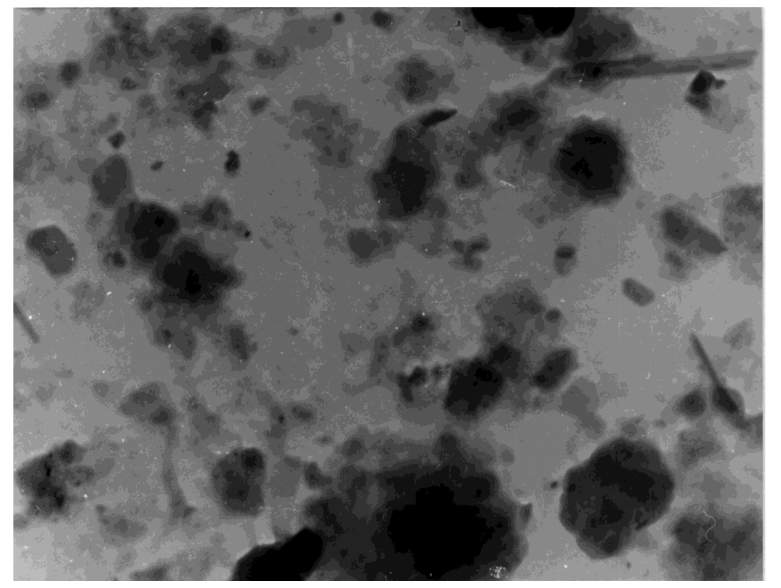

3

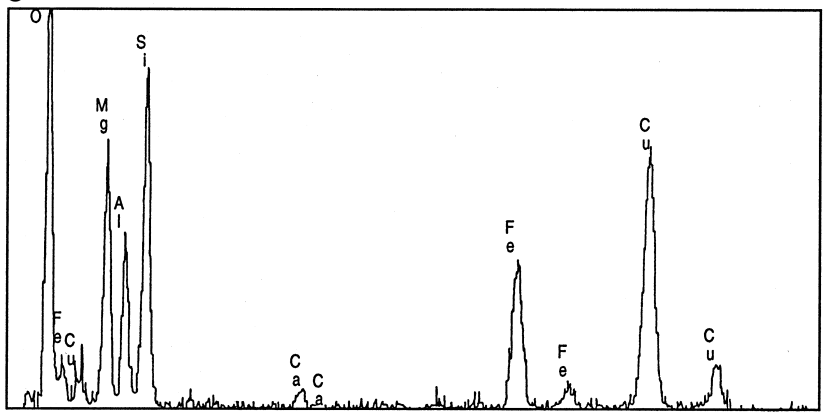

5

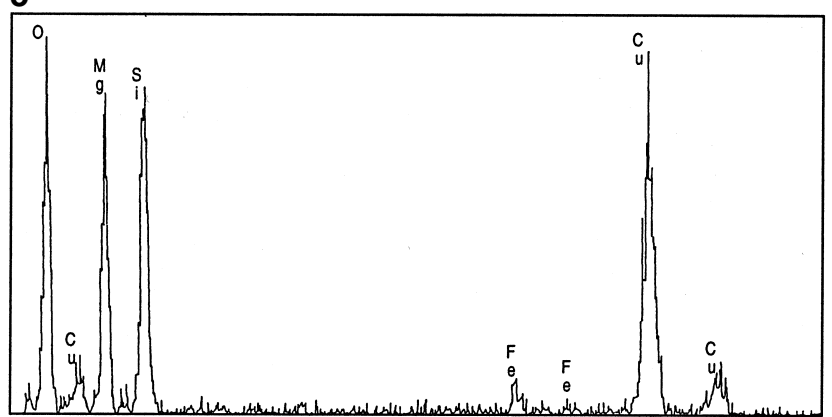

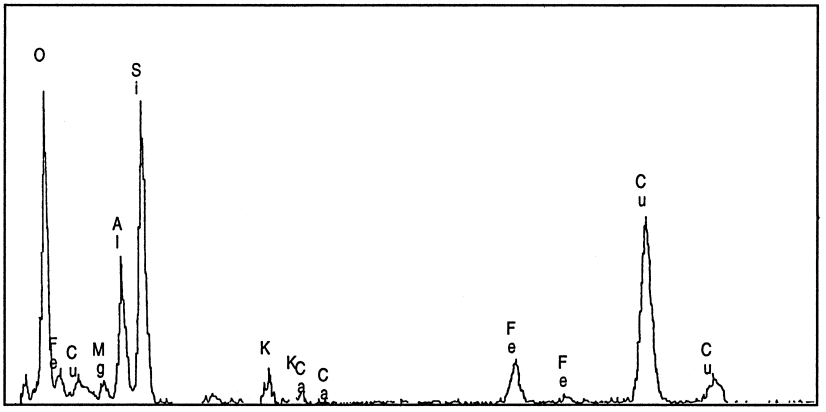

2

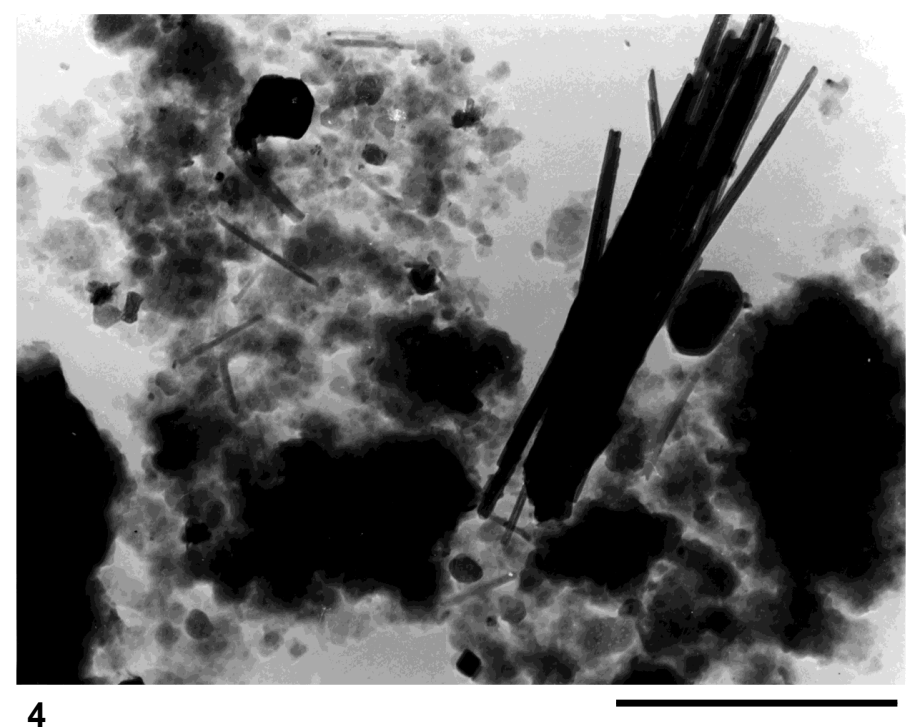

4

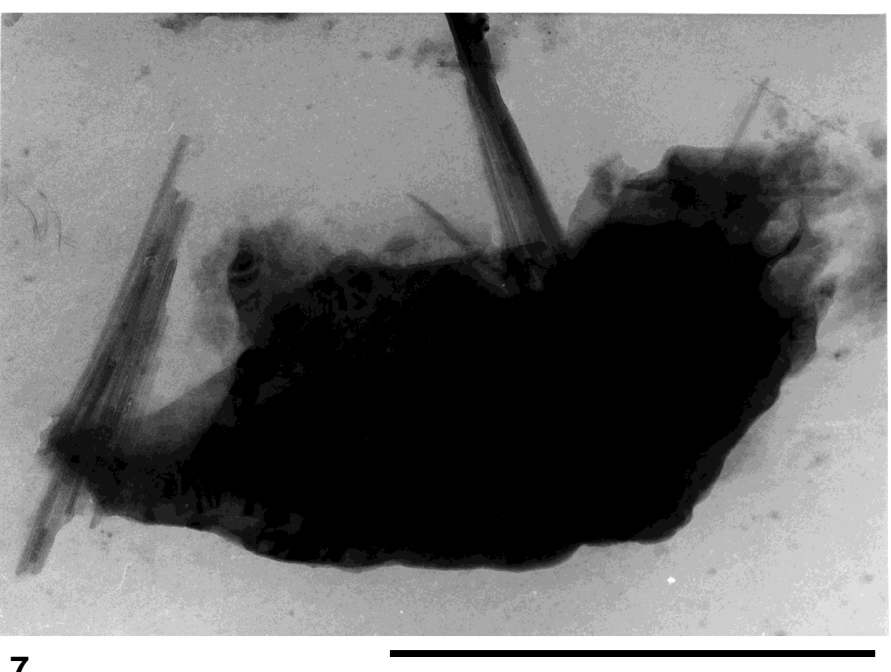

6

Plate 1. TEM photographs and elemental analysis of sediment particles by X-ray spectrometry. Bar $=1 \mu \mathrm{m}$. Cu in spectra corresponds to the copper grid used for the sample preparation. 1. Pliocene dark clay sample (Sample 160-968A-16X-3, 129-130 cm): a large flaky smectite and light small kaolinite. 2. Smectite spectrum from the large particle at the center of 1. 3. Pliocene dark clay sample (Sample 160-968A-16X-3, 129-130 cm): close-up of the light small kaolinite; the scattered fibers correspond to serpentine and palygorskite (from microprobe analysis). 4. Pliocene nannofossil ooze sample (Sample 160-968A-16X-4, 134-135 $\mathrm{cm}$ ): large smectite particles with some small-sized kaolinite, a bundle of serpentine minerals, and other small fibers (palygorskite?). 5. Fe-chlorite analysis of the big particle in 7. 6. Serpentine analysis of the fibers in 7. 7. Messinian sample (Sample 160-968A-25X-2, 55-56 cm): a large chlorite particle accompanied by serpentine fibers. 


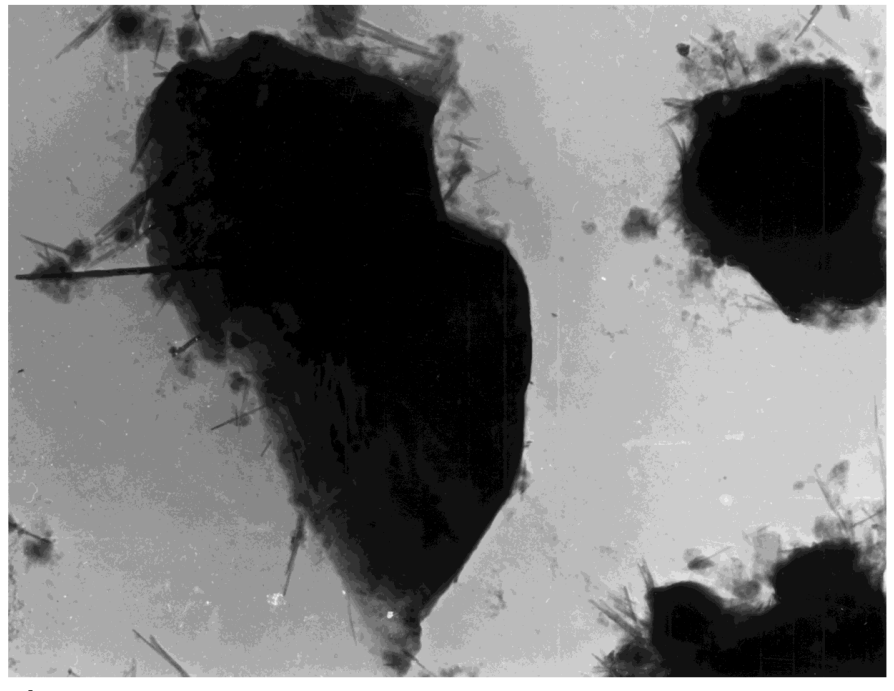

1

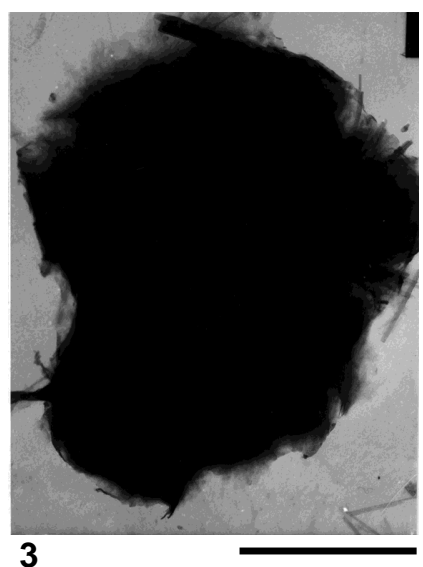

3
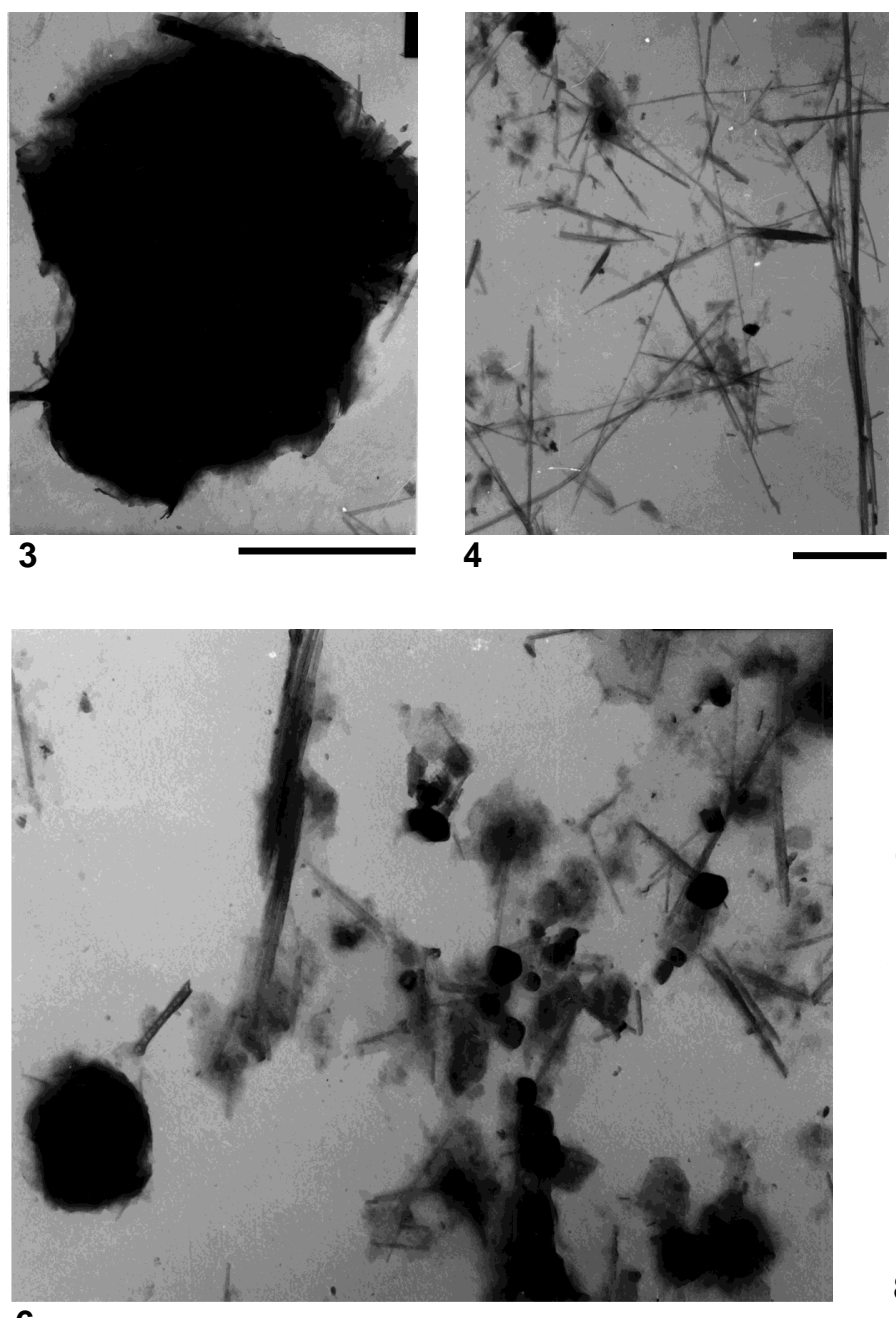

4
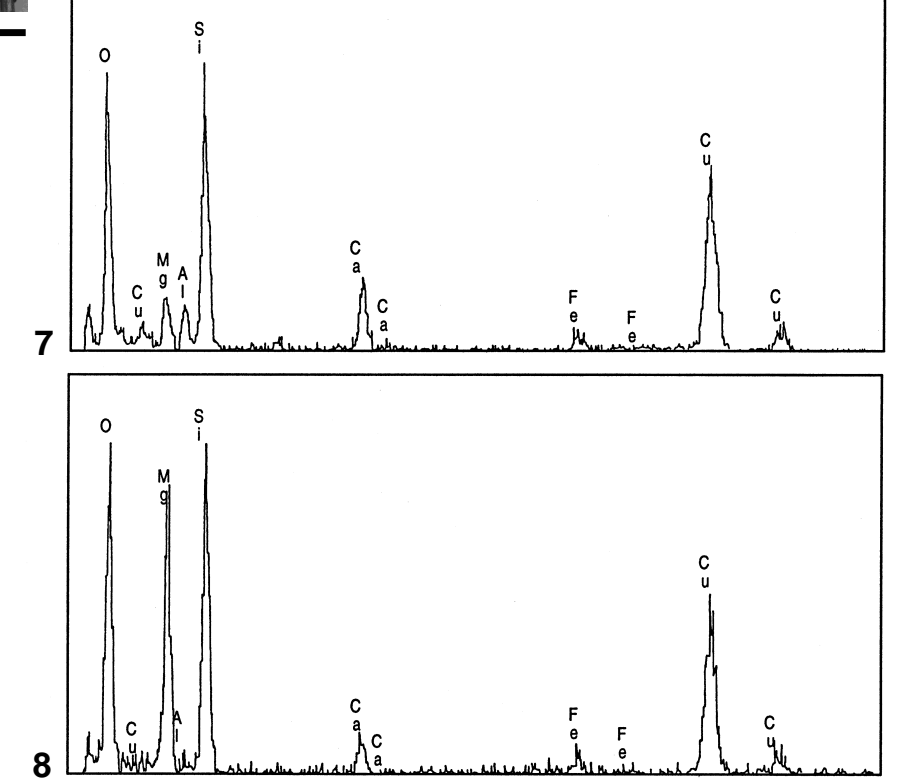

\section{6}

Plate 2. TEM photographs and elemental analysis of sediment particles by X-ray spectrometry. Bar $=1 \mu \mathrm{m}$. Cu in spectra corresponds to the copper grid used for the sample preparation. 1. Messinian sample (Sample 160-968A-25X-2, 55-56 cm): large chlorite (left), smectite, and small fibers (palygorskite + serpentine minerals). 2. Messinian sample (Sample 160-968A-27X-2, 30-31 cm): chlorite + smectite + fibers (palygorskite + serpentine minerals). 3. Messinian sample (Sample 160-968A-28X-6, 40-41 cm): smectite. 4. Messinian sample (Sample 160-968A-28X-6, 40-41 cm): palygorskite and serpentine minerals. 5. Analysis of palygorskite particle in 4. 6. Messinian sample (Sample 160-968A-33X-1, 35-36 cm): smectite + palygorskite and serpentine minerals. 7. Analysis of palygorskite particle in 6. 8. Analysis of serpentine particle in 6 . 


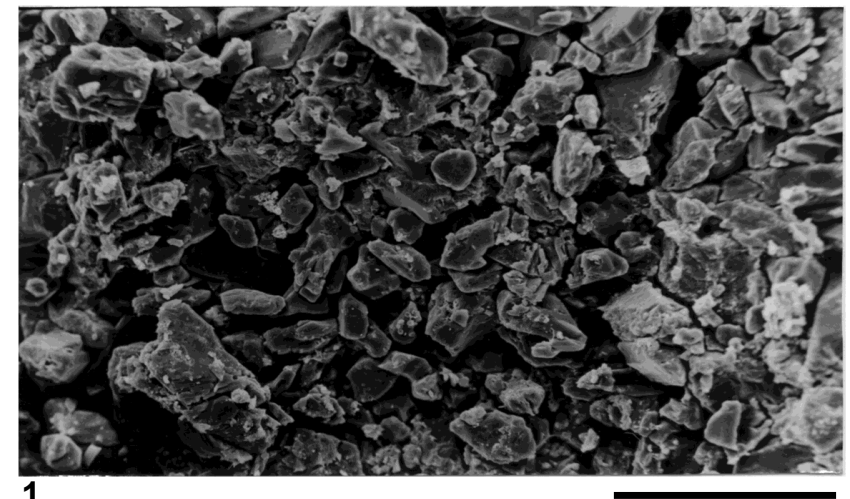

1

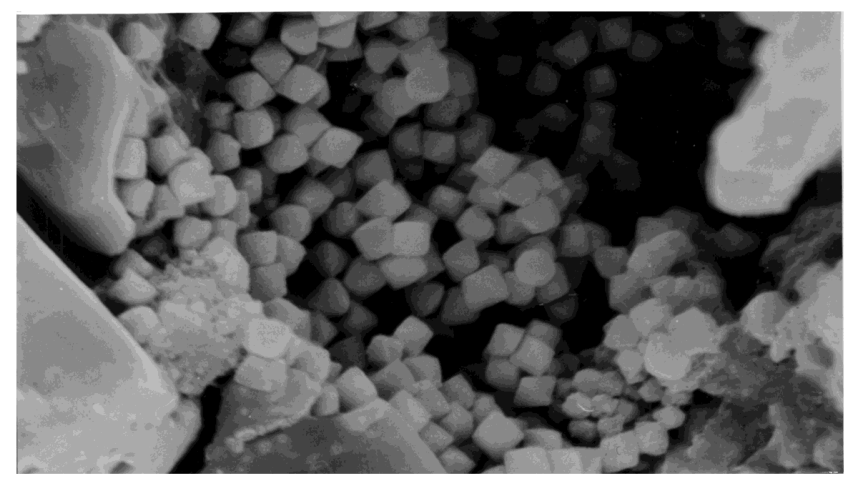

3
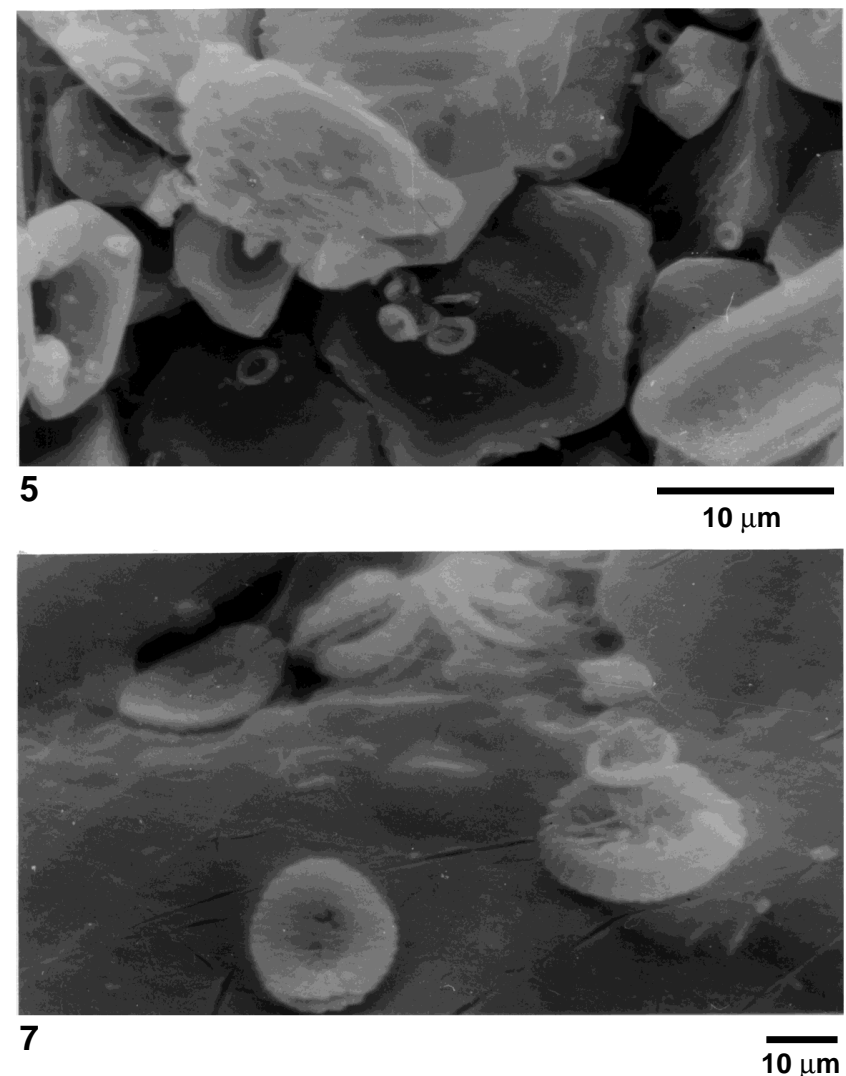

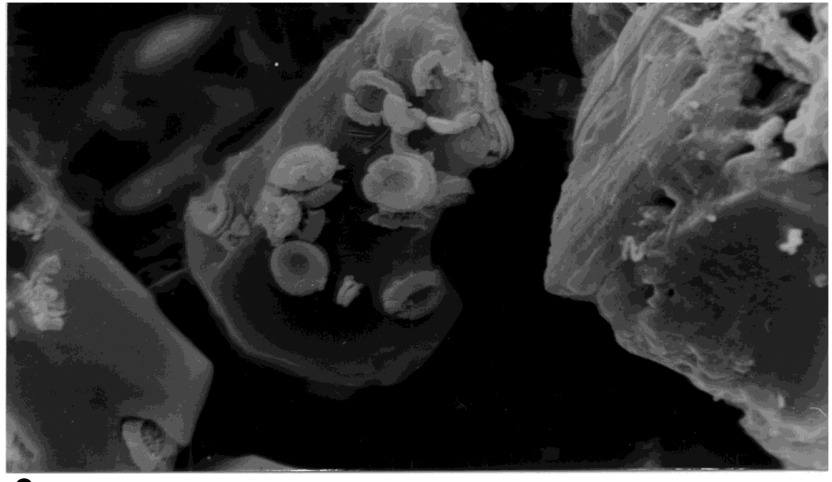

2

$10 \mu \mathrm{m}$

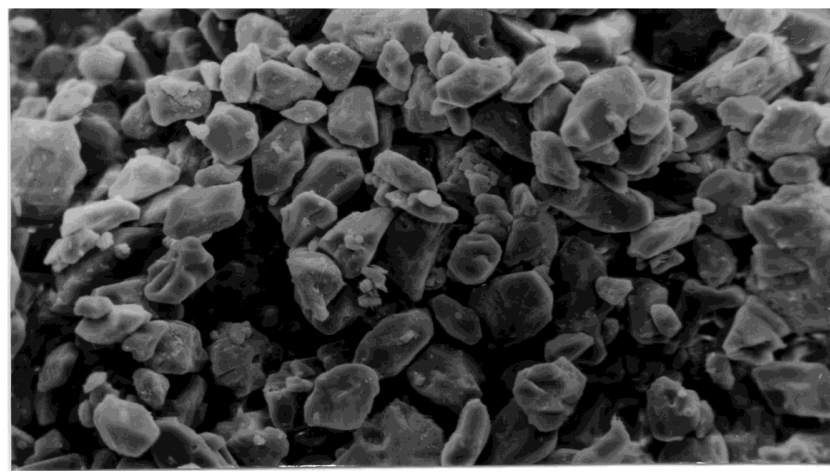

4

$50 \mu \mathrm{m}$

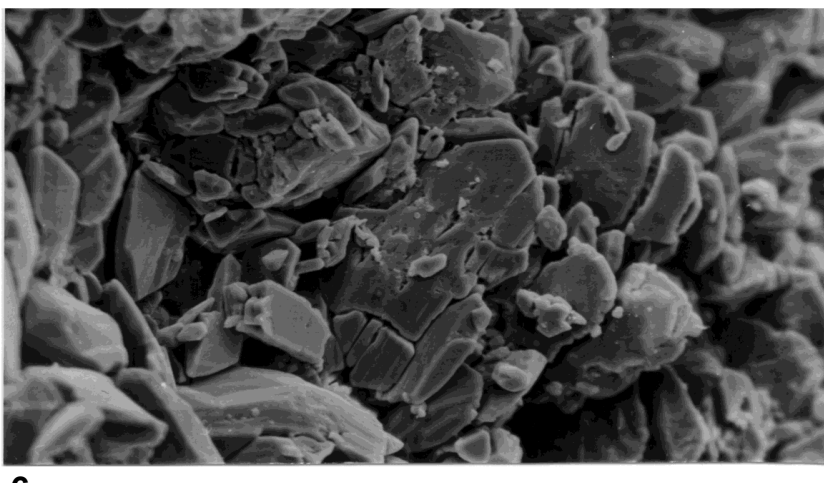

6

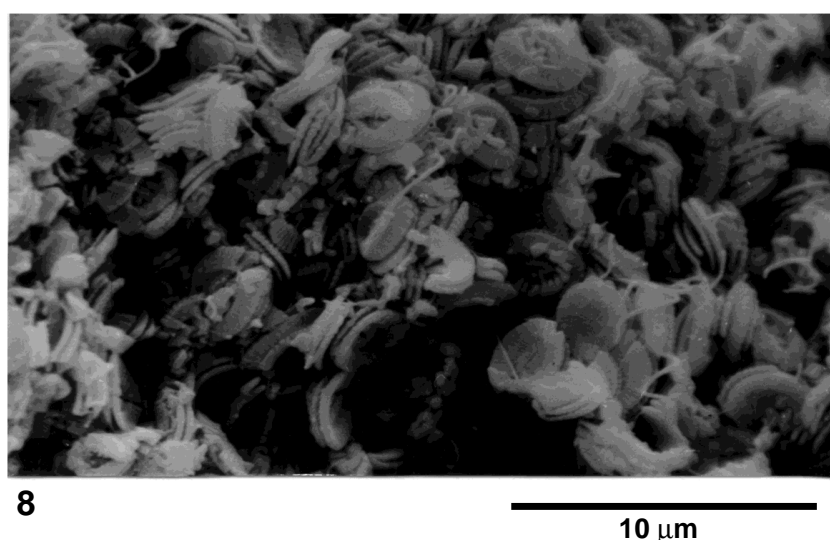

Plate 3. SEM photographs of Messinian Lago Mare gypsum samples. 1. Sample 160-968A-25X-2, 25-26 cm: heterometric gypsum crystals. 2. Sample 160968A-25X-2, 25-26 cm: close-up of gypsum crystals including monospecific (Reticulofenestra sp.) nannoplankton. 3. Sample 160-968A-25X-2, 25-26 cm: close-up of interlocking gypsum and pyrite (?) crystals. 4. Sample 160-968A-25X-2, 125-126 cm: gypsum crystals. 5. Sample 160-968A-25X-2, 125-126 cm: close-up of gypsum crystals including monospecific (Reticulofenestra sp.) nannoplankton. 6. Sample 160-968A-25X-CC, 27-28 cm: heterometric gypsum crystals. 7. Sample 160-968A-25X-2, 27-28 cm: close-up of gypsum crystals including monospecific (Reticulofenestra sp.) nannoplankton. 8. Sample 160-968A25X-2, 27-28 cm: intergypsum level of monospecific nannoplankton (Reticulofenestra sp.). 